\title{
Outbreak of Clostridium difficile-Associated Disease in a Small Animal Veterinary Teaching Hospital
}

\author{
J.S. Weese and J. Armstrong
}

\begin{abstract}
An apparent outbreak of enteric disease occurred in dogs and cats at a veterinary teaching hospital. Clostridium difficile Toxin A or B or both were identified in 1 or more fecal samples from 48 of $93(52 \%)$ dogs over a 5 -month period, 30 of which were identified in the 1 st 26 days, after which strict infection control measures, including closure to elective cases, were implemented. Affected animals included in-patients, out-patients that were housed temporarily in the wards, and resident blood donor dogs. Infection control measures, including partial depopulation, isolation, hospital and yard cleaning, and barrier precautions, were instituted, after which, the incidence of nosocomial diarrhea decreased from 19 cases per 1,000 admissions to 2.5 cases per 1,000 admissions $(P<0.001)$.
\end{abstract}

Key words: Diarrhea; Nosocomial; Clostridia.

$C^{l}$ ostridium difficile is a gram-positive anaerobic bacterium that has been implicated as a cause of enteric disease in humans and animals, including horses, dogs, cats,

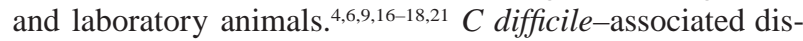
ease (CDAD) occurs as a result of intestinal colonization and toxin production by toxigenic strains of $C$ difficile. Strains of $C$ difficile can produce a number of toxins, but the enterotoxin designated Toxin A and the cytotoxin designated Toxin $\mathrm{B}$ are the best understood. CDAD is diagnosed on detection of these 2 toxins in feces. ${ }^{10}$ Not all strains of $C$ difficile produce toxins, and a small percentage of normal humans and animals can carry toxigenic strains without production of toxins and development of disease. ${ }^{10,16}$ Therefore, bacterial culture is not adequate for the diagnosis of CDAD, but it can be useful for epidemiologic purposes.

Outbreaks of CDAD are of concern in human hospitals and chronic care facilities. ${ }^{3,20,21}$ The role of $C$ difficile in nosocomial disease in veterinary hospitals is less well understood. An outbreak of CDAD involving 9 horses at a veterinary teaching hospital has been reported, ${ }^{11}$ but outbreaks of CDAD in dogs have not. This study describes investigation of an outbreak of enteric disease in the Ontario Veterinary College Small Animal Clinic (OVC-SAC).

\section{Materials and Methods Veterinary Hospital}

The OVC-SAC is a tertiary care referral center with approximately 9,000 canine admissions annually. Dogs are housed in 6 wards and an intensive care unit. Additionally, a colony of approximately 12 blood donor dogs is kept in a separate ward within the hospital. Ambulatory dogs are walked in an outdoor, fenced, grass-covered area that is connected to the hospital. Resident blood donor dogs are walked in an adjacent area. There is a common, gravel-covered entrance to these 2 areas.

From the Department of Clinical Studies (Weese) and the Veterinary Teaching Hospital (Armstrong), Ontario Veterinary College, University of Guelph, Guelph, ON Canada.

Reprint requests: J. Scott Weese, Clinical Studies, University of Guelph, Ontario Veterinary College, Guelph, ON N1G 2W1 Canada; e-mail: jsweese@uoguelph.ca.

Submitted February 11, 2003; Revised April 8 and June 17, 2003; Accepted July 14, 2003.

Copyright (C) 2003 by the American College of Veterinary Internal Medicine

0891-6640/03/1706-0007/\$3.00/0
Occasionally, diarrhea develops in hospitalized animals and resident dogs. An anecdotal increase in the frequency of diarrhea in resident dogs, followed by hospitalized dogs, was identified in April 2002, prompting an investigation.

\section{Case Definition}

Diarrhea was identified in client dogs and resident dogs by clinicians, technicians, kennel staff, or, in the case of animals that had been discharged, by owners or referring veterinarians. Cases were considered potentially nosocomial if they developed diarrhea between 48 hours after admission to the hospital and 14 days after discharge.

\section{Diagnostic Testing}

All fecal samples were tested for the presence of $C$ difficile Toxin $\mathrm{A}$ and $\mathrm{B}$ with a commercial enzyme-linked immunosorbent assay (ELISA). ${ }^{a}$ Selective culture for $C$ difficile was performed with both cycloserine-cefoxitin fructose agar (CCFA) and CCFA with $0.1 \%$ taurocholate. Other testing was performed at the discretion of the attending clinician and included $\mathrm{CBC}$, serum biochemistry, abdominal radiography, abdominal ultrasonography, fecal flotation and saline wet mount, Giardia antigen ELISA, ${ }^{\mathrm{b}}$ C perfringens enterotoxin (CPE) detection, ${ }^{\mathrm{c}}$ and selective culture for Salmonella, Yersinia, and Campylobacter spp. A random selection of fecal samples also was submitted for electron microscopy and virus isolation.

\section{Case Management}

The attending clinician directed management of individual cases. Metronidazole (15-20 mg/kg PO q12h) was administered to all $C$ difficile toxin-positive dogs. Treatment was prescribed for 7 days initially, but if $C$ difficile toxins were still present in the feces at the end of the initial treatment course or diarrhea had not resolved, metronidazole administration was continued for 10 more days. Resident dogs also were treated with di-tri-octahedral smectite (30 g PO q24h) and Lactobacillus rhamnosus strain $\mathrm{GG}^{\mathrm{e}}$ (40 billion colony-forming units PO q24h) and were provided with a high-fiber diet.

\section{Statistical Analysis}

Fisher's exact test was used for all comparisons. $P<0.05$ was considered significant.

\section{Results}

Fecal samples (181) were collected from 93 diarrheic dogs between April 3 and September 3, 2002. Fifteen dogs were resident animals, 7 were research animals, and 71 were privately owned. Samples (1-8; mean 1.85 , median 1) from each animal were evaluated. The age of affected 
Table 1. Laboratory information from Clostridium difficile toxin-positive and $C$ difficile toxin-negative diarrheic $\operatorname{dogs}(\mathrm{n}=93)$ during an apparent outbreak of enteric disease in a veterinary teaching hospital.

\begin{tabular}{lccc}
\hline & \multicolumn{2}{c}{$C$ difficile } & \\
\cline { 2 - 3 } & Toxin-positive & Toxin-negative & $P$ value \\
\hline $\begin{array}{l}\text { C difficile isolation } \\
C \text { perfringens } \text { entero- } \\
\text { toxin detection }\end{array}$ & $5 / 39(13 \%)$ & $4 / 38(11 \%)$ & 1.00 \\
$\begin{array}{l}\text { Giardia } \text { antigen } \\
\text { Other gastrointestinal }\end{array}$ & $1 / 29(7 \%)$ & $2 / 17(12 \%)$ & 0.62 \\
$\quad$ parasites \\
$\begin{array}{l}\text { Salmonella, Yersinia, } \\
\quad \text { or Shigella } \text { spp iso- }\end{array}$ & $1 / 17(6 \%)$ & $0 / 3$ & 1.00 \\
$\quad$ lation & $0 / 15$ & 1.00 \\
$\begin{array}{l}\text { Camplylobacter } \text { spp } \\
\text { isolation }\end{array}$ & $1 / 6(17 \%)$ & $0 / 11$ & \\
\hline
\end{tabular}

${ }^{a}$ As detected by fecal smear and flotation.

animals ranged from 1 to 18 years $(5.7 \pm 3.7$, mean \pm $\mathrm{SD})$. Overall, $C$ difficile toxins were detected in 1 or more fecal samples from 48 of 93 (52\%) dogs. Seven of the 48 (15\%) dogs developed CDAD after discharge from the hospital. Co-infection with other enteropathogens was identified uncommonly in $C$ difficile toxin-positive dogs (Table 1). Electron microscopy and virus isolation were performed on fecal samples from 21 randomly selected dogs (11 from CDAD dogs and 10 from non-CDAD dogs). Potentially clinically relevant findings were present in 2 dogs. A suspected enterovirus of unknown consequence was identified in a dog with CDAD and a possible coronavirus was identified in the feces of a dog without CDAD.

No significant differences $(P<0.05)$ in the proportion of dogs undergoing surgery or receiving antimicrobial drugs or chemotherapy were found between dogs with a diagnosis of CDAD and those without. On the basis of the timing of hospitalization and clinical signs, nosocomial infection was suspected in 38 of 48 (79\%) CDAD and 31 of 45 (69\%) non-CDAD dogs. Only 9 CDAD dogs and 8 nonCDAD dogs had been treated with antimicrobials before the onset of diarrhea. Small sample size precluded analysis of risks associated with different antimicrobials. Only 2 dogs died or were euthanized. Both had CDAD, but death was from unrelated causes in $1 \mathrm{dog}$. In the 2 nd dog, euthanasia was performed because of severe hemorrhagic gastroenteritis and septicemia. Hemorrhagic gastroenteritis was diagnosed in 1 other dog. This dog also tested positive for $C$ difficile but responded to treatment. No other pathogens were identified in either dog with hemorrhagic gastroenteritis.

Metronidazole was administered for $>7$ days in 9 dogs ( 2 client dogs and 7 resident dogs) because feces were still $C$ difficile toxin-positive or diarrhea was still present after the initial treatment period. Fecal samples were collected from 20 dogs after treatment. The time from initiation of treatment to resolution of clinical signs and negative fecal toxin ELISA was variable, ranging from 1 to 15 days (mean 7.6, median 6, SD 4.7). However, follow-up information was most readily obtained from resident dogs, which an-

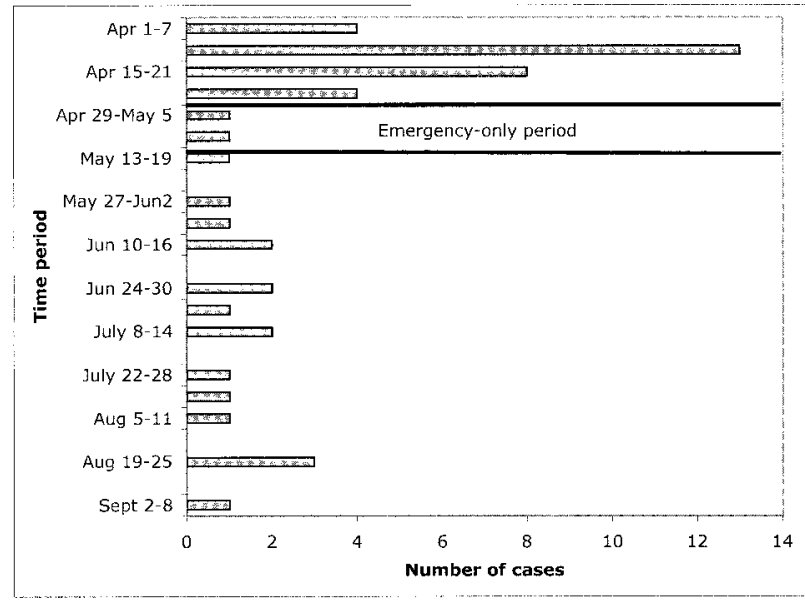

Fig 1. Temporal distribution of cases of Clostridium difficile-associated diarrhea in an apparent outbreak of disease at a veterinary teaching hospital $(\mathrm{n}=48)$.

ecdotally appeared to take longer to respond to treatment than client-owned dogs. Additionally, fecal samples were not necessarily collected on the 1st day after resolution of clinical signs. Many clients reported that their dogs were clinically normal within 48 hours of initiation of treatment, and compliance with requests for further fecal samples was poor.

After recognition of an increased number of cases of diarrhea in hospitalized and resident dogs, additional infection control measures were instituted, including quarantine of resident animals, implementation of full barrier precautions (eg, gowns, gloves, disposable overboots) when handling resident dogs or entering their housing area, emphasis on personal hygiene, frequent cleaning of floor surfaces with $10 \%$ bleach solution, and placement of disinfectant footbaths at the entrance to the intensive care unit and teaching animal ward. A scheduled emergency-only period, set to coincide with the transition between university semesters, was expanded so that elective cases were not admitted for 14 days. Additionally, less critical emergencies were directed to other facilities whenever possible. The dog walking yard was thought to be a potential source of infection because it was a common area that most infected animals had visited, because there was a high likelihood of fecal contamination of the grass and soil, and because teaching dogs also were turned out in an adjacent area. Approximately $20 \mathrm{~cm}$ of topsoil was removed and replaced with clean topsoil and sod. A significant decrease $(P<$ 0.0001 ) in cases of CDAD occurred after implementation of these measures. During the 26-day period from recognition of the 1st case until partial hospital closure, 30 cases of CDAD were identified, whereas 18 cases were identified in the next 136 days (Fig 1). A similar decrease in suspected nosocomial cases of CDAD also occurred. Seventeen cases of suspected nosocomial CDAD in client animals out of 893 total canine and feline admissions were identified during the initial period, corresponding to a nosocomial CDAD rate of 19 cases in 1,000 admissions. In the period after the clinic was reopened, 10 cases of nosocomial CDAD were reported out of 3,930 total admissions, with a 
nosocomial CDAD rate of 2.5 in 1,000 admissions $(P<$ $0.001)$.

\section{Discussion}

This study identified a cluster of cases of enteric disease in a small animal referral hospital, many of which were associated with $C$ difficile. An outbreak of CDAD of this scale has not been reported previously in dogs. The possibility that $C$ difficile was not the primary cause of disease was considered. Co-infection with another enteropathogen was uncommonly identified, but only a limited number of samples were tested for the presence of other enteropathogens. Although it is impossible to differentiate hospital-acquired infections from hospital-expressed, community-acquired infections or from infections acquired at referring veterinary clinics, the cluster of cases was suggestive of an outbreak of nosocomial CDAD. A number of possible sources of nosocomial $C$ difficile must be considered. $C$ difficile can be present in the gastrointestinal tract of a small percentage of normal dogs, cats, and people., , $7,8,12,13,16$ One or more animals might have been actively shedding $C$ difficile, but direct animal-to-animal transmission is unlikely because no direct contact occurred among hospitalized animals. Hospital personnel could have acted as fomites, passing $C$ difficile among patients or from the environment to patients.

It is suspected that infection was acquired from the hospital environment in this situation. Despite being poorly aerotolerant in its vegetative form, $C$ difficile can form spores that are highly resistant to disinfectants, ${ }^{19}$ and $C$ difficile spores have been detected in the environment of our veterinary hospital and others. ${ }^{1,14}$ Bleach is the most effective environmental disinfectant for $C$ difficile, but it is uncommonly used as a routine disinfectant in veterinary hospitals, in part because it is noxious and caustic. Numbers of $C$ difficile organisms might have increased over time as resistant spores accumulated after being shed by symptomatically and asymptomatically infected animals. These spores then could infect susceptible animals, particularly those receiving antimicrobials or chemotherapeutic agents. It is curious, therefore, that this outbreak started in the group of resident dogs and that intermittent cases of CDAD have continued to occur in these dogs. These dogs are housed in the teaching hospital but have no direct contact with patient animals. A potential source of infection, however, is possible because these dogs walk through parts of the hospital that house clinical patients. They are walked in an area directly adjacent to where clinical patients are walked, and these areas share a common entrance. Previously, we demonstrated that this entrance was heavily contaminated with $C$ difficile spores. ${ }^{14}$ On the basis of our understanding of nosocomial $C$ difficile in humans, the resident dogs could have been considered at somewhat lower risk because they had not received antimicrobials, had no history of diet changes or dietary indiscretion, and lived in a stable environment. The reason the resident dogs were affected is not clear, but their lifestyle could be more stressful than is recognized, and they might be exposed continually to $C$ difficile in the environment, creating a possible cumulative effect. One resident dog continued to experience recurrent episodes of CDAD for approximately 6 months and was adopted by a private party. No additional episodes of diarrhea were reported in this dog. It was also unexpected and alarming that some client dogs developed CDAD after discharge and after spending only a few hours in the hospital. The significant decrease in nosocomial CDAD after institution of aggressive infection control procedures suggests that these measures were effective in controlling this apparent outbreak; however, this suggestion is impossible to prove. Such infection control measures are logical on the basis of current understanding of $C$ difficile in veterinary and human medicine and should be considered if further outbreaks of CDAD are encountered in veterinary hospitals.

$C$ difficile was isolated from only a small number of animals with or without diarrhea. This finding was not surprising because $C$ difficile is a strict anaerobe that does not survive well in fecal samples, whereas the toxins are quite stable. ${ }^{15}$ Bacterial culture was not performed immediately in all cases because toxin detection is the clinical standard for diagnosis of CDAD. Additionally, once it was recognized that CDAD was the cause of a number of cases of diarrhea, metronidazole often was administered at the 1st sign of clinical disease, sometimes before obtaining a fecal sample. These factors likely contributed to the low isolation rate. Unfortunately, the low isolation rate precluded molecular epidemiologic studies.

Hemorrhagic gastroenteritis (HGE) was present in 2 dogs from which $C$ difficile toxins were isolated. It is unclear whether $C$ difficile was the primary cause or whether isolation represented secondary overgrowth. Severe hemorrhagic enteritis caused by $C$ difficile has been reported in other species, and Cave et $\mathrm{al}^{5}$ reported a significant association between detection of $C$ difficile toxins and acute hemorrhagic diarrheal syndrome in dogs.

Interestingly, despite the large number of cases of CDAD in the hospital wards and what would be considered a highly susceptible population in the intensive care unit (ICU), no cases of nosocomial CDAD were identified in the ICU. The reason for this finding is unclear, but it might relate to standard infection control measures in the ICU that are more stringent and uniformly enforced compared with the general wards. Additional infection control measures for the ICU were instituted, including restrictions on the personnel entrance into the ICU, walking ICU dogs in a separate area, collecting feces on paper plates so that no environmental contamination occurred, and placing a footbath containing $10 \%$ bleach solution at the entrance to the unit.

$C$ difficile might be an overlooked pathogen in small animal veterinary practice. Commercial ELISA testing for $C$ difficile toxins is becoming more widely available, and considering that $C$ difficile has been implicated as an important cause of diarrhea in dogs presented to primary care veterinary clinics, ${ }^{17}$ as well as a cause of nosocomial diarrhea, it would be prudent to incorporate $C$ difficile toxin testing into standard testing protocols.

\section{Footnotes}

${ }^{a}$ Clostridium difficile TOX A/B Test II, TechLab Inc, Blacksburg, VA b ProSpecT Giardia Microplate Assay, Alexon-Trend, Ramsey, MN 
c Clostridium perfringens enterotoxin test, TechLab Inc, Blacksburg, VA

${ }^{d}$ BioSponge, Platinum Performance, Buelton, CA

${ }^{\mathrm{e}}$ Culturelle, CAG Functional Foods, Omaha, NE

\section{Acknowledgment}

This study was supported by the Ontario Veterinary College Pet Trust.

\section{References}

1. Berry AP, Levett PN. Chronic diarrhoea in dogs associated with Clostridium difficile infection. Vet Rec 1986;118:102-103.

2. George RH, Symonds JM, Dimock F, et al. Identification of Clostridium difficile as a cause of pseudomembranous colitis. Br Med J 1978;1(6114):695.

3. Jones RL, Adney WS, Shideler RK. Isolation of Clostridium difficile and detection of cytotoxin in the feces of diarrheic foals in the absence of antimicrobial treatment. J Clin Microbiol 1987;25:12251227.

4. Weese JS, Staempfli HR, Prescott JF. A prospective study of the roles of Clostridium difficile and enterotoxigenic Clostridium perfringens in equine diarrhoea. Equine Vet J 2001;33:403-409.

5. Weese JS, Staempfli HR, Prescott JF, et al. The roles of Clostridium difficile and enterotoxigenic Clostridium perfringens in diarrhea in dogs. J Vet Int Med 2001;15:374-378.

6. Weese JS, Weese HE, Bourdeau TL, et al. Suspected Clostridium difficile associated diarrhea in two cats. J Am Vet Med Assoc 2001; 218:1436-1439.

7. Worsley MA. Infection control and prevention of Clostridium difficile infection. J Antimicrob Chemother 1998;41(Suppl C):59-66.

8. Lyerly DM, Neville LM, Evans DT, et al. Multicenter evaluation of the Clostridium difficile TOX A/B test. J Clin Microbiol 1998;36: 184-190.
9. Bender BS, Bennett RG, Laughton BE, et al. Is Clostridium difficile endemic in chronic-care facilities? Lancet 1986;2:11-13.

10. Wilcox MH, Smyth ETM. UK survey of the incidence and impact of Clostridium difficile infection 1993-96. Programme of the Third Federation of Infection Societies Conference 1997; Abstract P20: (abstract).

11. Madewell BR, Tang YJ, Jang S, et al. Apparent outbreak of Clostridium difficile-associated diarrhea in horses in a veterinary teaching hospital. J Vet Diagn Invest 1995;7:343-346.

12. Barbut F, Corthier G, Charpak Y, et al. Prevalence and pathogenicity of Clostridium difficile in hospitalized patients: A French multicentre study. Arch Int Med 1996;156:1449-1454.

13. George WL, Rolfe RD, Mulligan ME, et al. Infectious diseases 1979-Antimicrobial agent-induced colitis: An update. J Infect Dis 1979;140:266-268.

14. Groschel DHM. Clostridium difficile infection. Crit Rev Clin Lab Sci 1996;33:203-245.

15. Perrin J, Buogo C, Gallusser A, et al. Intestinal carriage of Clostridium difficile in neonate dogs. Zentralbl Veterinarmed 1993;40: 222-226.

16. Weber A, Kroth P, Heil G. The occurrence of Clostridium difficile in fecal samples of dogs and cats [in German]. Zentralbl Veterinarmed 1989;36:568-576.

17. Wilcox MH, Fawley WN. Hospital disinfectants and spore formation by Clostridium difficile. Lancet 2000;356:1324.

18. Al-Saif N, Brazier JS. The distribution of Clostridium difficile in the environment of South Wales. J Med Microbiol 1996;45:133137.

19. Weese JS, Staempfli HR, Prescott JF. Isolation of environmental Clostridium difficile from a veterinary teaching hospital. J Vet Diagn Invest 2000;12:449-452.

20. Weese JS, Staempfli HR, Prescott JF. Survival of Clostridium difficile and its toxins in equine feces: Implications for diagnostic test selection and interpretation. J Vet Diagn Invest 2000;12:332-336.

21. Cave NJ, Marks SL, Kass PH, et al. Evaluation of a routine diagnostic fecal panel for dogs with diarrhea. J Am Vet Med Assoc 2002;221:52-59. 